\title{
Der Luganersee. Betrachtung zu einem Brief des Humanisten Francesco Cicereio aus Nailand an den Luganeser Arzt Girolamo Camuzio aus dem Jahr 1559
}

\author{
Von Charles Salzmann, Zürich
}

Kartographen und Historiker des 16. Jahrhunderts schienen es mit ihren Landschaftsaufrissen und mit geschichtlichen Annalen nicht allzu genau zu nehmen, wie dies vor allem auf alten Landkarten so leicht ins Auge fällt. Verzeichnungen und Verzerrungen des Kartenbildes mögen sich reizvoll darbieten in den einfachen frei erfundenen Linien, unbeschwert, ungemessen, fein säuberlich und etwas steif ausgeführt; weil ihnen der Gedanke an die Raumgestaltung entging, blieben ganze Landschaften vergessen. Bei Historikern spielten andere Gründe eine oft greifbare Rolle, wie politische Rücksichten, persönliche Verpflichtungen, aber auch lässige Unkenntnisse und Mißachtungen.

Einen solchen Verstoß leistete sich um die Mitte des 16. Jahrhunderts kein geringerer als der einflußreiche Höfling, Arzt, Historiker und Bischof von Nocera, Paolo Giovio ${ }^{2}$, Bürger von Como, der den Comersee (Lario) in so lobseligen Worten gepriesen, daß er darob den nachbarlichen Luganersee (Ceresio) vergaß. Seiner Beschreibung des Lario gab er eine Karte bei, auf welcher er den Luganersee, wie Cicereio im Briefe angibt, "auf eine so schäbige Winzigkeit verkleinerte, daß er ihn verächtlicher nicht hätte zeichnen können». (atque in ea adjectum esse vestrum istum lacum, tam in parvum et angustum locum conclusum, nihil ut majori cum contemptu exprimi potuerit).

Diese geographische Geringschätzung seines heimatlichen Ceresio vermochte Francesco Cicereio nicht zu verwinden; es kränkte ihn, daß ein so hoher Herr, dazu ein Nachbar aus Como und päpstlicher Historiker, den unvergleichlichen Luganersee überging.

In einem Brief an seinen Freund Girolamo Camuzio ${ }^{3}$ in Lugano, voll Liebe zum alten Borgo Lugano bewußt eidgenössisch fühlend, forderte er diesen auf, vereint mit den Freunden Caesar Castoriuss ${ }^{4}$ und Giuseppe Ossutius $^{5}$, Akten zu sammeln, damit ihm eine gediegene Beschreibung der Landschaften um den Luganersee gelinge.

Cicereio schreibt: Wenn schon der Gardasee, der Benacus, in Liedern einst von Dulcinus Secundus ${ }^{6}$ und Pietro Bembo ${ }^{7}$ gefeiert wurde 
(Valerius Catull ${ }^{8}$ und P. Vergilius $\mathrm{Maro}^{9}$ übergeht er), der Lario, der Comersee nun in Giovio seinen Verherrlicher gefunden habe und selbst vom Verbano (Lago Maggiore) eine Schilderung erwartet werde ${ }^{10}$, so sei es an der Zeit, sich des Luganersees zu erinnern.

Diese Literatur über die oberitalienischen Seen kannte Cicereio gewiß genau. Bei seiner Liebe zur heimatlichen Erde vermöge er es kaum zu ertragen, daß diese liebliche Gegend so wenig gewürdigt werde. Er sei zwar nicht der Mann, der sich ohne anderer Mithilfe zutraue, ihr Lob zu singen, aber bereit, Aufzeichnungen zu sammeln für ein solches Werk mit der Unterstützung Girolamo Camuzios und seiner Freunde. Im Geiste sah er schon, wie ein solches Werk Gestalt annehmen könne. Dies ergebe sich beim Betrachten der herrlichen Landschaft, mit den Rebbergen, den üppigen Fruchtfeldern in ihrer Vortrefflichkeit, der Zahl der Ortschaften mit ihren begabten Einwohnern, als auch der Stadt, die Hervorragendes biete mit ihrer angesehenen Statthalterschaft. Es gebe zwar Leute, welche dieser Gegend jede Vergangenheit absprechen, da sie erst seit kurzem aufgeblüht sei.

Cicereio erinnerte sich hier der Entwicklung Luganos ${ }^{11}$ nach der Errichtung der eidgenössischen Landvogtei.

Angeregt durch seine humanistischen Studien, suchte er eifrig nach römischen Altertümern, Inschriften und Münzen in Oberitalien; unlängst hatte ihm Girolamo Camuzio das Epigraph der römischen Familie der Negro dei Negri aus der Chiesa di San Pietro zu Gravesano im Val d'Agno vermittelt ${ }^{12}$.

Ferner werde eine alte Inschrift eines Columella ${ }^{13}$ gezeigt in der Vorhalle des St.-Martin-Heiligtums zu Campione.

Und da behaupten gewisse Leute, diese Gegend sei erst in neuerer Zeit stark besiedelt, und halten sie nicht für altes Kulturgebiet. Er besitze zwei Münzen aus der Hand Ossutrús', eine griechische in Silber, die man einst aus tiefstem Wurzelwerk der Kastanienbäume bei Fontana ${ }^{14}$ ausgegraben, und eine römische, die sich beim Ausheben der Fundamente für eine Kirche auf dem Monte Bremio fand.

Damit war er sich der früheren Romanitas des Luganese voll bewußt.

Augustino Pianta ${ }^{15}$ berichtete ihm, sein Schwiegervater Giacomo FerRARIO $^{16}$ besitze öffentliche Urkunden, in denen manches von Sonvico ${ }^{17}$ und der Umgebung überliefert werde, die aus der Zeit des Langobardenkönigs Desiderius stammen, was auch Aegidius Tschudi ${ }^{18}$ bestätigt habe.

In Bironico ${ }^{19}$ sei ferner ein Bündel staatlicher Akten vorhanden, Lugano betreffend. 
Den zehnjährigen Krieg (1118-1127) zwischen Mailand und Como gedenke er ins rechte Licht der Geschichte zu rücken, jenes mittelalterliche Ringen um die Kastelle am See, zwischen Guelfen und Ghibellinen, als die kommunalen Freiheiten erstritten wurden ${ }^{20}$.

Freudehell begeistert überschaut Cicereio in Gedanken das Seegestade: est enim in animo a ripa inchoare et primo sinistrum latus perlustrare, wo sein glückliches Lugano liegt - in cuius medio felici sydere Luganum positum.

Als Lugano Sitz einer eidgenössischen Landvogtei wurde, erlebte das Seestädtchen einen ansehnlichen Aufschwung ${ }^{21}$.

Von der Terrasse über der Stadt leuchtet jetzt die edle Fassade von San Lorenzo, um 1517 in spätgeborener Gestalt der Frührenaissance errichtet. Im alten Borgo erhoben sich nun Palazzi mit Portalen, Innenhöfe mit Galerien, weite Kaufhäuser und draußen im Grünen zwischen Rebgelände und Gärten Landhäuser - insigni cultu, quotdie magis, atque magis ornatur pulcherrimisque aedificiis augetur.

Dann schweift sein Blick ins Valle Luganese, wo des Freundes Landhaus steht mit dem Castello (Trevano) ${ }^{22}$, von Schriftstellern hochgepriesen (villa tua, cum arce scriptoribus aliquot celebrata), von da nach Castagnola und einer endlosen Reihe von Dörfern (inde pene infinita alia, quae nunc commemorandi non est locus), bis er Porlezza erreicht (ubi Porliciam applicuero).

Gegen Osteno auf dem rechten Seeufer (ad dextrum latus me convertam et statim occurret mihi Ostenum) werde er neben anderen berühmten Männern den bekannten Dichter Antonius Leucus ${ }^{23}$ erwähnen (ubi praeter caeteros viros illustreis [sic], poeta non ignobilis Antonius Le UCus mihi erit celebrandus).

Mit beredten Worten wolle er die einsam bewaldeten diesseitigen Höhen mit den gegenüberliegenden fruchtbaren Berghängen vergleichen, wie auch den Aufenthaltsort der Wasservögel schildern (quae a mergorum statione nomen ${ }^{24}$ habet) und ebenso die Abhänge des Monte Caprino (montibus caprinis describendis, locis sterilis) und die Uferlandschaften von Campione, Bissone, Maroggia bis hinauf zum Monte Generoso beschreiben.

Dem allem gedenke er eine Abhandlung voranzustellen, die Art und Gestalt des ganzen Sees erklären solle. Die westlich gelegenen Seearme, den Lago d'Agno, die Gegend von Ponte Tresa erwähnt er nicht. Girolamo Camuzio und sein Bruder Andreas mögen ihm behilflich sein, damit ihm nichts entgehe, was ihm an natürlichen Gegebenheiten allenfalls unbe- 
kannt sei. Alle ihre Unterlagen seien ihm zur Einsichtnahme vonnöten, damit er nicht erst eine Reise unternehmen müsse. Er forderte sie auf, ihm zunächst einmal ihren Bestand an alten Akten zu senden, was sie ja leicht und ohne größeren Aufwand tun könnten, damit er die notwendigen Auszüge mache, um sie hernach zurückzusenden.

Vorerst gedenke er, die Kunstdenkmäler zu bearbeiten, wozu bereits von Augustino Pianta Vorarbeit geleistet sei, denen er weitere hinzufüge, wenn sie von solchen berichten könnten.

Genaue Angaben wünsche er über historische Ereignisse, die erinnerungswürdig, aber kurz gefaßt sein müßten, Dinge, die sie selbst erlebt haben oder aus der Überlieferung kennen. Wenn er ihnen dadurch etwas Arbeit auflade, so mögen sie bedenken, daß keine hervorragende Leistung entstehe ohne gemeinsame Anstrengung. Er selbst habe bei dieser großen Arbeit keine andere Absicht, als diesen Ceresio, seine Vaterstadt und die ehrenwerte Statthalterschaft zu ehren.

Was immer ich schreiben werde, soll nicht meinem, sondern Euerem Urteil unterstehen, wobei ich hoffe, daß Du vor allem mir helfen werdest, diese Arbeit zu glätten und auszufeilen.

VI. Kal. Septembr. MDLIX Mediol.

Lebewohl!

(26. September 1559 Mailand)

Diesem Briefe Cicereios an Girolamo Camuzio und die Luganeser Freunde kommt etwelche Bedeutung zu, weil er den vielversprechenden Anlauf zur ersten Naturbeschreibung des Luganersees enthält.

An einer rein literarischen Lobeshymne lag ihm wohl kaum, eher an einer auf wissenschaftlich gesichtetem Material fundierten, wohldokumentierten geographischen, historischen, wirtschaftlich-ökonomischen, kulturellen und künstlerischen Darstellung für die Gebildeten und vor allem für die Signori Svizzeri.

Wäre die Arbeit Francesco Cicereios damals im Druck erschienen, was für ein vortreffliches Quellenwerk über Lugano und die Landschaften am Ceresio wäre späteren Generationen geschenkt worden! Aber schon so birgt der Brief als Quelle manche geographische, geschichtliche, biographische und kulturelle Andeutung.

Wenn auch bis um die Jahrhundertwende von 1600 der Luganersee auf Landkarten in zu kleiner Gestalt und vollständig unrichtig dargestellt wurde, war er doch schon damals den Luganesen wie den Svizzeri stets lieb; 
so grüßte ihn Heinrich Glarean (1488-1563) im Helvetiae Panegiricon (Coloniae 1512) mit den folgenden Worten:

Quid memorem antiquum dulci memorabile cantu

Lucanum a luce aut Lucanis nomine dictum,

Romano propius Como, lacuique minori,

Parte Cisalpina, quo non locupletius ullum est.

In diesem Brief leuchtet zum erstenmal der Gedanke der Verbundenheit eines berühmten Luganeser Humanisten mit der alten Eidgenossenschaft auf; im Zeichen der diesjährigen Zentenarfeier werden sich Tessiner und die übrigen Schweizer dieser so langen Verbundenheit mit besonderer Freude erneut bewußt sein.

${ }^{1}$ Francesco Cicereio, von Lugano, 1521 (Motta), 1527 (Casati, Oldelli) bis 1596; auch Ciceri, Cicerino, Cesarino, wie er sich in Briefen nannte. Er studierte in Lugano bei Giovanni Menabene: Latein, Griechisch, Mathematik, Dialektik; eine Zeitlang wirkte er auch als Lehrer in Lugano. Seit 1554 weilte er als Lateinlehrer und Humanist in Mailand, wo er 1561 zum Professor am Lyceo aufstieg. Mit Cardinal Carlo Borromeo, Girolamo Cardano, Marcantonio Maioragio, Joh. Oporinus, Andrea Camuzio, dem damaligen Landvogt Hieron. Frick von Bern stand er in freundschaftlichem Briefwechsel, der zum Teil Ponpeo Casatı in zwei Bänden 1782 veröffentlichte; dieser Sammlung wurde dieser Brief entnommen. Der Verfasser war etwa zweiunddreißig Jahre alt, als er ihn schrieb. - Poмpeo Casati, Francesci Cicereii epistolarum libri XII. Mediolani 1782, Tom. II. Epistola XXXIII, p. 78-80. - G. A. OLdelli, Dizionario storico-ragionato delli uomini illustri del Canton Ticino. Lugano 1807, p. 66-71 und p. 9, Fußnote. Scrittori della Svizzera italiana. Vol. I, Bellinzona 1936, p. 9-22. Bollettino storico della Svizzera italiana: 1881, 1887, 1890, 1902. - C. Cantù, Storia della città e della diocesi di Como. Como 1831, Vol. II, p. 332. - L. Brentani, Miscellanea storica ticinese. Como 1926, Vol. I., p. 242, 243, 369, 370. - HBL, Bd. 2, p. 588.

2 Paolo Giovio (Iovius), von Como, 1483-1552, studierte in Como, Mailand, Padua und Pavia, wo er den Doktor Medizinae erwarb. In Rom arztete er in Gelehrtenkreisen, wo er bald Anerkennung bei Papst Leo X. fand, der ihm die Professur der Rhetorik in Rom anvertraute. Daneben machte er sich einen Namen als Historiker seiner Zeit (1494-1547). Guicciardini und MachiavelLi bezeichneten ihn als den bedeutendsten Historiker in der ersten Hälfte des 16. Jahrhunderts. Basler Offizinen druckten fünf seiner Geschichtswerke. Mit dem Papste Clemens VII., der ihn zum Bischof von Nocera erhöhte, reiste er zur Krönung KARLS V. nach Bologna 1530. Der Kaiser empfing ihn in feierlicher Audienz. An Heinrich II. von Frankreich schrieb er: "Io ho già temperata la penna d'oro col finissimo inchiostro.» Mit der Wahrheit in seinen Historiographien scheint er es nicht immer allzu genau zu halten. Der Papst betraute ihn oft mit schwierigen Gesandtschaften, so zu Pietro Aretino nach Venedig. Neben seinen vielen geschichtlichen Arbeiten führte P. Grovio ein bewegtes Leben. Die Beschreibung des Lario von P. Giovio erschien als posthumes Werk in Mailand 1558. - Pauli Iovir, Descriptio 
Larii lacus. In Historiarum sui temporis. Basileae 1578. - Dictionaire des sciences médicales. Biographie médicale. Paris 1822, Tome 5: P. Giovio, p. 372-373. - Enciclopedia italiana, Vol. 17: P. Grovio. - J. Burckhardt, Kultur der Renaissance in Italien. Berlin 1927 : P. Giovio. - C. Cantù, Storia della città e della diocesi di Como. Como 1831, Vol. II, P. Giovio: p. 147-155. - v. C. Chledowski, Rom. Die Menschen der Renaissance. München 1922, P. Giovio: p. 280-284.

${ }^{3}$ Grolamo Camuzio, von Lugano, Bruder des Andrea Camuzio, mit dem er 1549 am Religionsgespräche in Locarno teilnahm.

${ }^{4}$ Caesar Castorius, von Lugano, Castoreo, Castoreus, Castorea, Castoyra, altes, heute erloschenes ghibellinisches Geschlecht. HBL, Bd. 2, p. 517.

${ }^{5}$ Giuseppe Ossutius (Ossuzio), von Lugano, Advokat in Lugano, 1571-1581. - G. Martinola: Guida dell'Archivio cantonale. Bellinzona 1951, p. 77. - G. A. Oldelli, Dizionario storico ragionato degli uomini illustri del Canton Ticino. Lugano 1807, p. 50.

${ }^{6}$ Dulcinus Secundus, aus Sirmione, Dichter eines Carmina, das von Catull und dem Benacus handelt, gewidmet Jacobus Antonius Turriano, erschienen in Mailand 1502 (Casati, Vol. II, p. 81).

7 Pietro Bembo, 1470-1547, venetianischer Patrizier, Notar der römischen Kurie; Leo X. erhob ihn zum Grafen, Paulus III. zum Kardinal. Lebte seit 1520 in Padua seiner literarischen Muse. - Seine Opere, 12 Vol. Milano 1808-1810. - G. M. MazzuccheldI, Gli scrittori d'Italia. Brescia 1760, Vol. II, p. $733 \mathrm{ff}$.

${ }^{8}$ C. Valerius Catullus, geb. 84. v. Chr. in Verona. Es sei an die Verse erinnert: Paene insularum, Sirmio, insularumque...

${ }^{9}$ P. Vergilius Maro, aus Andes bei Mantua, 70-19 v. Chr. Verse aus Georgica II, Verse 159-160, den Larius betreffend: anne lacus tantos? te, Lari maxime teque, fluctibus et fremitu adsurgens Benace marino?

${ }^{10}$ Domenico Macaneo, auch Domenico della Bella, Domenico Belli, il Macagno genannt, geboren 1438 in Macagno am Verbano, gestorben achtzigjährig in Turin. Verfasser der Verbani lacus locorumque adjacentium chorographica descriptio. Mediolani 1490. - Eine Ausgabe erschien 1690 mit Zusätzen von Lazzaro Augustino Cotta.

${ }^{11}$ Lugano war schon während des Mittelalters ein wichtiger Marktplatz, wo alljährlich im Herbst ein großer Viehmarkt stattfand : fiera di calend setembar. - Wie bedauernswert es um Lugano und das Valle Luganese stand, als 1499 französische Soldtruppen das Castello besetzt hielten, vernimmt man aus: M. DE MAUlde - LA-Clavière, La conquète du Canton du Tessin par les Suisses ..., p. 29: «Lugano est le plus pauvre lieu et les pyres gens que j'aie jamais vus les gerfes (Guelfen) ont battu, brûlé cette ville, qui est presque toute gerfelyne. Pour or ni pour argent, on n'y trouve de blé pour le château ni pour nous, car les paysans, qui ont fait ce déluge, ont tout emporté aux montagnes et si je n'étais arrivé, Lanse au poin s'en allait, n'y pouvant plus vivre» (aus dem Rapport des Kommandanten Jacques de Leyve). - Franco Paravicini, Beitrag zur Rechtsgeschichte des Luganese, unter Comaskischer, Mailändischer und Eidgenössischer Herrschaft. Diss. Zürich, Poschiavo 1934, p. 8, 50. - Mit der Besetzung Luganos durch die Eidgenossen 1513 zog ein neuer Geist ein, der die Stadt aufleben ließ. - Lugano zählte um die Mitte des 16. Jahrhunderts etwa 1500-2000 Seelen.

${ }^{12}$ L. A. Muratori, Thesaurus veterum inscriptionum. Vol. 6, Mediolani 1737-1743, Tom. I, p. 294: «C. Gemino Nigro VI. Vir. / C. Geminius C. F. Niger / Patri.» - G. A. Oldeldi, Dizionario ... Lugano 1807, p. 161, Fußnote. - E. PoMetTA, Lugano dall'epoca romana 
al 1400. Archivio storico della svizzera italiana. Anno III. No. 1-2 (Gennaio-Giugno) 1928, p. 4. - E. Pometta, Saggi di storia ticinese dall'epoca romana alla fine del medio evo. Bellinzona 1930, Vol. I, p. 19. - E. МоттA e S. Ricci, Il Luganese nell'epoca preromana e romana. Pubbl. Dipartimento della pubblica Educazione del Ct. Ticino, o. Jahr, p. 48, 78, 84. - E. Howald und E. Meyer, Die römische Schweiz. Zch. 1940, p. 194. - A. CRIvelli, Atlante preistorico e storico della Svizzera italiana. Bellinzone 1943, Vol. I, p. 94.

${ }^{13}$ L. Junius Moderatus Columella, aus Gades, im 1. Jahrhundert. Verfasser De re rustica, Libri XII. Lugduni 1548. In diesen Büchern lobte er die Weine Oberitaliens. Vermutlich steht er in keiner Beziehung zur Inschrift.

14 Fontana bei Paradiso-Lugano. HBL, Bd. 3, p. 192.

15 Augustino Pianta (Planta, del Pianta), von Lugano, 1511 bis 7. März 1572. Er starb im Alter von einundsechzig Jahren (OLdelli, p. 139). Dr. iur., bedeutender Jurist und Humanist, Advokat in Lugano; Freund Cicereios, Hieron. Fricks und Aegidius Tschudis. - Oldelli, Dizionario, p. 138-140. - L. Brentani, Miscellanea storica ticinese. Como 1926, Vol. I, p. 176, 242-244-HBL, Bd. 5, p. 432.

${ }^{16}$ Giacomo Ferrario, Capitano des Gian-Giacomo Medici in Florenz. Seit 1533 Richter zu Sonvico. - Oldelli, Dizionario, p. 85.

17 Sonvico: Der Curtis Sonvico schenkte 724 der Langobardenkönig Luitprando der Basilica di San Carpoforo in Como. Diese Urkunde gilt als Fälschung. - P. Schaefer, Das Sottocenere im Mittelalter. Affoltern am Albis 1931, Diss. Zürich, p. 198, Fußnote ${ }^{250}$. G. Rovelli, La castellanza di Sonvico. Massagno 1927, p. 22.-HBL, Bd. 6, p. 449, 450. ${ }_{18}$ Aegidius Tschudi, von Glarus, 1505-1572.

${ }^{19}$ Bironico: Hier stand ein Castello der Rusca zur Sicherung des Monte Cenere; erstmals 1205 genannt, wurde es 1418 unter den Visconti vergrößert.- HBL, Bd. 2, p. 254.

${ }^{20}$ Geschichtlich war man bis 1926 nur wenig orientiert über das Sottocenere; EMiLio MоттA veröffentlichte seit $1879 \mathrm{im}$ «Bollettino storico della Svizzera italiana» viel Archivmaterial, das keine weiten Übersichten erlaubte. Erst die Arbeiten von MaspoLI, Brentani und vor allem Schaefers erbrachten umfassendere Darstellungen. - E. MASpoli, La Pive di Agno. Memorie storiche. Como 1917. - L. Brentani, Miscellanea storica ticinese. Como 1926. - P. SchaEfer, Das Sottocenere im Mittelalter. Ein Beitrag zur Geschichte der Südschweiz und des italienischen Mittelalters. Diss. Zürich, Affoltern am Albis 1931.

${ }^{21} \mathrm{Zu}$ Anfang des Mittelalters war Lugano zwischen den umgebenden Höhen eine bescheidene Siedlung. Das Marktrecht stand bereits im 9. Jahrhundert dem Bischof von Como zu. Die weitere Entwicklung begünstigten die Humiliaten mit ihrer Wollindustrie im 13. Jahrhundert. Ende des 14. Jahrhunderts bereisten Luganeser Tuchhändler die Märkte von Locarno und Lags im Bündner Oberland (Schaefer, p. 34), und um die Mitte des 14. Jahrhunderts lassen sich Färbereien nachweisen. In den dreißiger Jahren des 16. Jahrhunderts belebten die Welser aus Augsburg (Bartolomeo et socii) die Textilmanufactur von neuem. - L. Brentani, Miscellanea storica ticinese. Como 1926 (Welser). Vol. I, p. 314, 323, 324.

22 villa tua, cum arce scriptoribus celebrata: damit bezeichnet Cicereio fraglos das Landhaus des Girolamo Camuzio, das vermutlich beim Castello Trevano lag, während das Castello San Michele ob Casserate damals bereits zerfallen (Schaefer, p. 93-94), Fußnote ${ }^{131}$ - F. Ballarini, Croniche di Como. Compendio delle croniche della città di Como. Como 1619, Par. 3 , p. 306. «Il castello della valle d'Intellvo è opera de' CAmutiI, già 
conti di detta valle fabbricato negli anni 1260.» Später auch das Castello der Quadri genannt. - A. L. TatTi, Annali sacri di Como. Como e Milano 1663-1683.

${ }^{23}$ Antonius Candidus (griechisch Leucus) Decembrius, lebte im 15. Jahrhundert. F. Argelati, Bibliotheca scriptorum mediolanensium. Milano 1745. Vide Antonius Leucus, p. 2100.

${ }^{24}$ Giovanni Anastasi, Il lago di Lugano. 2. Auflage. Lugano 1926, p. 155. Der Name «statione mergorum» hat sich in der Flurbezeichnung Sasso mergone bis zu Anfang des 19. Jahrhunderts in Karten erhalten. - Vermutlich handelt es sich bei den Wasservögeln, die Cicereio nennt, um die heute noch auf dem See sich in dieser Gegend tummelnden «Gabbianelli», eine Möwenart. 